\title{
VIRAL INFECTION
}

\section{How SARS-CoV-2 makes the cut}

The spike protein of SARS-CoV-2 harbours a cleavage motif for host cell proteases that is not found in closely related viruses. Peacock and colleagues show that this motif allows the virus to evade innate antiviral defences and is required for transmission.

\section{Markus Hoffmann and Stefan Pöhlmann}

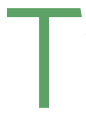
he coronavirus disease 2019 (COVID-19) pandemic, caused by severe acute respiratory syndrome coronavirus 2 (SARS-CoV-2), has devastated societies, health systems and economies. Despite intense research, mechanisms of pathogenesis are incompletely defined, including the biological significance of the $\mathrm{S} 1 / \mathrm{S} 2$ cleavage site in the viral spike protein (S). Reporting in this issue of Nature Microbiology, Peacock and co-authors show that cleavage at $\mathrm{S} 1 / \mathrm{S} 2$ in the SARS-CoV-2 S protein (SARS-2-S) enables evasion of innate antiviral factors, the interferon-induced transmembrane proteins (IFITM), and is required for transmission in a ferret model ${ }^{1}$.

Entry of coronaviruses into host cells depends on activation of the viral $S$ protein by host cell proteases, which involves cleavage at two sites, named S1/S2 and S2: The sequence of the S1/S2 cleavage motif in SARS-2-S is unique among related coronaviruses. Unlike the S1/S2 motifs of SARS-CoV-related viruses, which mostly contain a single basic residue, the sequence of the SARS-2-S S1/S2 motif harbours several basic residues (R-R-A-R). Of note, 'multi-basic' cleavage motifs are usually found in the haemagglutinin proteins of highly pathogenic avian influenza A viruses. In addition, the $S$ proteins of some non-SARS-CoV-related coronaviruses, including human coronavirus HKU1 (R-R-K-R-R), harbour multi-basic motifs. However, whilst multi-basic, these cleavage motifs are not closely related to the one present in SARS-2-S.

Initial studies revealed that the S1/S2 site is required for entry of SARS-CoV-2

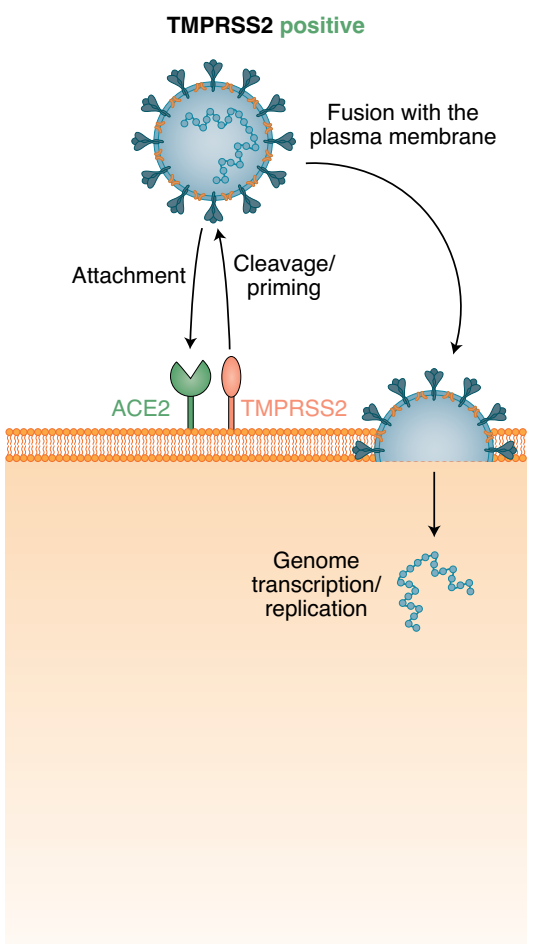

b

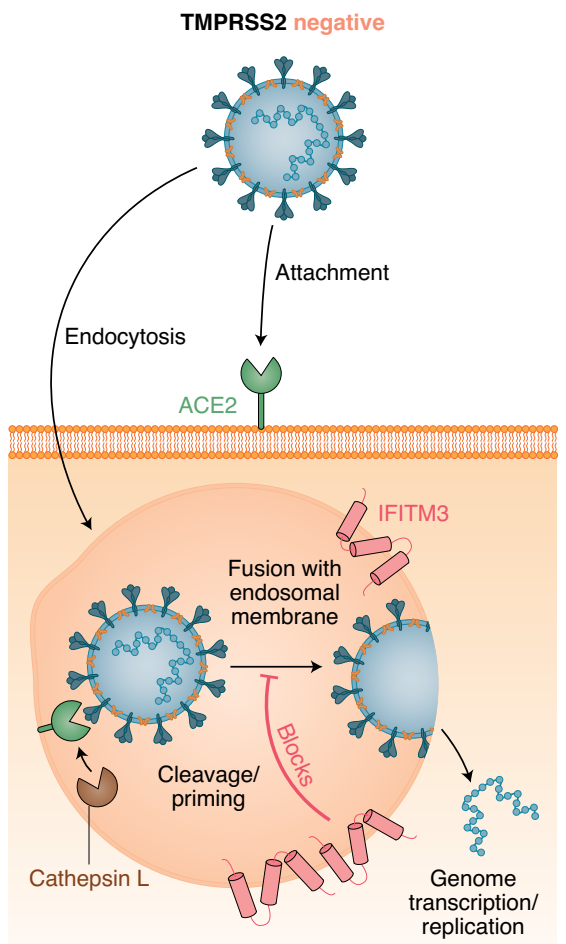

Fig. 1 | Effects of SARS-CoV-2 S1/S2 cleavage on replication and transmission. a, Upon receptor binding, SARS-2-S is activated by the serine protease TMPRSS2 and the viral envelope fuses with the plasma membrane. $\mathbf{b}$, In cells lacking TMPRSS2, SARS-CoV-2 enters cells by endocytosis and the S protein is cleaved by cathepsin L to drive fusion between viral and endosomal membranes. The cellular interferon-induced antiviral transmembrane protein IFITM3 inhibits the endosomal entry route (b) but has no effect on the cell surface entry route (a). c, An intact S1/S2 site is required for efficient viral amplification in the upper respiratory tract and transmission of SARS-CoV-2 in a ferret model. $\Delta$ CS, S1/S2 cleavage site removed; WT, wild-type S1/S2 cleavage site. 
into human lung cells. The S1/S2 site is cleaved by the proprotein convertase furin in lung cells, and cleavage is required for $S$ protein activation by the cellular serine protease transmembrane protease serine 2 (TMPRSS2), and for viral entry ${ }^{2}$ (Fig. 1). However, these initial findings were obtained with surrogate systems and have not been confirmed with authentic SARS-CoV-2 lacking the S1/S2 site. Further, it was unknown whether an intact S1/S2 site was required for viral transmission between individuals. Finally, it was unclear how SARS-CoV-2 benefited from TMPRSS2 usage (facilitated by cleavage at $\mathrm{S} 1 / \mathrm{S} 2$ ), given that another S-protein-activating protease, cathepsin L, was identified in animal and human cell lines and is broadly expressed.

Peacock and colleagues used recombinant SARS-CoV-2 to show that an intact S1/S2 site is required for SARS-CoV-2 infection of TMPRSS2-positive human cell lines and primary human airway epithelial cells (which also express TMPRSS2) ${ }^{1}$. They also show that entry into TMPRSS2-negative cell lines is mediated by cathepsin $\mathrm{L}$, an endosomal/lysosomal cysteine protease known to cleave and activate coronavirus $S$ proteins in certain cell culture systems. Using amphotericin B treatment and overexpression of IFITM3, they show that activation of the $S$ protein by TMPRSS 2 at the cell surface allows SARS-CoV-2 to evade the antiviral activity of IFITM proteins, which constitute antiviral restriction factors located at the cell surface (IFITM1) or at the endosome/lysosome (IFITM2 and IFITM3 $)^{2}$. Finally, they prove that an intact S1/S2 site is required for efficient viral amplification in the upper respiratory tract and for transmission of SARS-CoV-2 in a ferret model (Fig. 1).

The finding that efficient SARS-CoV-2 spread in airway epithelial cells relies on the integrity of the S1/S2 site, and therefore on $\mathrm{S}$ protein activation by TMPRSS2, is consistent with other studies ${ }^{3-5}$. What advantage is there in spatially and temporally separating cleavage at the S1/ S2 and S2' site? Both SARS-CoV and SARS-CoV-2 depend on TMPRSS2 for lung cell entry, but SARS-2-S is cleaved at S1/S2 during $S$ protein biogenesis in infected cells and at S2' during viral entry into target cells, whereas SARS-CoV S protein is most likely processed at both sites during cell entry. One explanation is that cleavage at the S1/S2 site during $\mathrm{S}$ protein biogenesis enables robust viral spread via cell-cell fusion ${ }^{4}$.

The study by Peacock and colleagues provides a compelling explanation for why coronaviruses exploit TMPRSS2 for S protein activation. Activation by TMPRSS2 removes the need to enter the endosome/ lysosome and therefore enables evasion of the antiviral factors IFITM2 and IFITM3. These proteins block endosomal/lysosomal entry of SARS-CoV-2 and other viruses at the membrane fusion step - an effect that can frequently be rescued by the antifungal drug amphotericin $\mathrm{B}^{2,6}$, although the underlying mechanism is incompletely understood.

Inhibition of SARS-CoV-2 entry by IFITM proteins and TMPRSS2-dependent rescue has also been observed in other studies ${ }^{7,8}$, and it has been demonstrated that IFITM2 is crucial for inhibition of SARS-CoV-2 infection by type I interferon ${ }^{8}$. The postulated TMPRSS2-dependent evasion of IFITM proteins via cleavage at the $\mathrm{S} 1 / \mathrm{S} 2$ site is likely important for viral spread in and between hosts. The finding that SARS-CoV-2 lacking the $\mathrm{S} 1 / \mathrm{S} 2$ site is attenuated in mice ${ }^{9}$ and hamsters ${ }^{9,10}$ is consistent with the reduced viral load that Peacock et al. observe in the nasal epithelium of ferrets, and the inefficient transmission among ferrets is likely due to reduced titres.

Peacock et al. found that the SARS-CoV-2 wild type did not outcompete the cleavage-site-defective virus in the nasal epithelium of co-infected animals, which is puzzling. It will be interesting to examine whether spread in nasal epithelium was entirely TMPRSS2 dependent, since several TMPRSS2-related enzymes have been shown to activate SARS-2-S and are expressed in the upper respiratory tract ${ }^{3}$. Finally, it should be noted that although TMPRSS2 promotes viral spread in the respiratory epithelium, amplification of SARS-CoV-2 in extrapulmonary organs may be independent of TMPRSS2.
Future studies with mice lacking IFITM $2 / 3$ and/or TMPRSS 2 should address whether TMPRSS2-mediated evasion from restriction factors occurs in vivo. Similarly, it will be interesting to compare IFITM expression levels in pulmonary and extrapulmonary cells since this may reveal the existence of target cell subsets with low IFITM $2 / 3$ expression and thus low requirement for TMPRSS2 for viral entry. Further, the effect of IFITM polymorphisms on the course of SARS-CoV-2 infection should be studied, since IFITM3 single-nucleotide polymorphisms affect the severity of influenza A virus infection in humans.

Exploiting a cell surface protease such as TMPRSS2 for S protein activation, rather than an endosomal/lysosomal protease such as cathepsin L, may enable SARS-CoV-2 to evade innate antiviral restriction factors. In turn, this immune evasion tactic enables replication to high titres in the respiratory tract, thereby maximizing virus transmission.

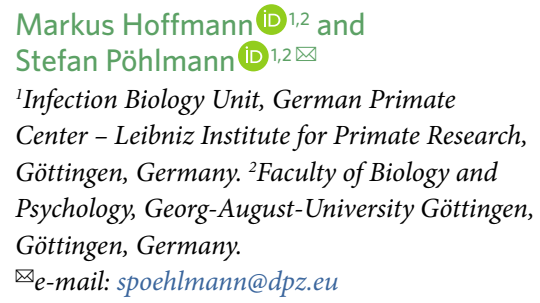

Published online: 30 June 2021

https://doi.org/10.1038/s41564-021-00931-x

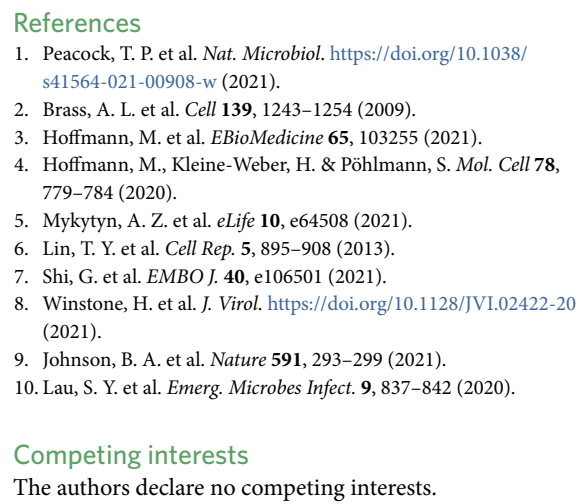

